\title{
FUNGOS MICORRÍZICOS ARBUSCULARES EM RIZOSFERAS DE TRÊS ESPÉCIES DE FITOBIONTES INSTALADAS EM ÁREA DE MATA CILIAR REVEGETADA
}

\author{
Rosilaine Carrenho ${ }^{1}$ \\ Sandra F. B. Trufem ${ }^{2}$ \\ Vera L. R. Bononi ${ }^{2}$
}

Recebido em 12/4/2000. Aceito em 22/9/2000

\begin{abstract}
RESUMO - (Fungos micorrízicos arbusculares em rizosferas de três espécies de fitobiontes instaladas em área de mata ciliar revegetada) Com o objetivo de avaliar o comportamento das espécies de fungos micorrízicos arbusculares (FMA) estabelecidas em áreas revegetadas de mata ciliar, foram coletadas amostras de solo rizosférico de Croton urucurana Baill., Inga striata Willd. e Genipa americana L. Estas plantas são indígenas em matas ciliares, sendo a primeira considerada pioneira, a segunda, secundária inicial e a terceira, espécie clímax. Vinte e duas espécies de FMA foram identificadas. O maior número de espécies foi observado em rizosferas de $C$. urucurana e $I$. striata (15 espécies), enquanto o maior número de esporos foi observado em rizosferas de $G$. americana (511 esporos/100g solo). Glomus apresentou o maior número de espécies (10), seguindo-se de Acaulospora (6), Scutellospora (4), Gigaspora e Entrophospora (cada com uma espécie). Quanto à frequiência das espécies, G. macrocarpum Tul. \& Tul. ocorreu em maior número de amostras em Croton; G. claroideum Schenck \& Smith, G. etunicatum Becker \& Gerd., G. macrocarpum e G. occultum Walker predominaram em Genipa, e E. kentinensis Wu \& Liu, G. etunicatum e G. macrocarpum, em Inga. Concluiu-se que o número de esporos de FMA tende a aumentar com o estádio sucessional, enquanto os índices diversidade, riqueza e equabilidade de espécies de Glomales tendem a diminuir nas comunidades estabelecidas nas rizosferas da espécie clímax.
\end{abstract}

Palavras-chave - Glomales, mata ciliar, revegetação, estádio seral

\begin{abstract}
Arbuscular mycorrhizal fungi in rhizospheres of three phytobionts established in a revegetated riparian area) To evaluate the specific composition of arbuscular mycorrhizal fungi (AMF) established in recomposed areas of gallery forest, samples of rhizospheric soil were collected from Croton urucurana Baill., Inga striata Willd. and Genipa americana $\mathrm{L}$. These plants are indigenous in gallery forests, being the first considered as pioneer, the second as early secondary and $G$. americana as climax, according to the successional stages. Twenty-two species of AMF were identified. The highest richness was observed in rhizospheres of $C$. urucurana and $I$. striata (15 species), while the highest number of spores occurred in $G$. americana rhizospheres ( 511 spores $/ 100 \mathrm{~g}$ dry soil). Glomus showed the highest number of species (10), followed by Acaulospora (6), Scutellospora (4), Gigaspora and Entrophospora (each with one species). The most frequent species in Croton was G. macrocarpum Tul. \& Tul.; in Inga, E. kentinensis Wu \& Liu, G. etunicatum Becker \& Gerd. and G. macrocarpum; and in Genipa, G. claroideum
\end{abstract}

\footnotetext{
Universidade Estadual de Maringá, Departamento de Biologia, Av. Colombo 5790, CEP 87090-900, Maringá, PR, Brasil
} Instituto de Botânica, Seção de Micologia e Liquenologia, C. Postal 4005, CEP 01061-970, São Paulo, SP, Brasil 
Schenck \& Smith, G. etunicatum, G. macrocarpum and G. occultum Walker. We concluded that the number of spores increases with the successional stages; conversely, the indices of diversity, richness and equability diminish in the rhizosphere of climax plants.

Key words - Glomales, gallery forest, revegetation, seral stage

\section{Introdução}

Fungos micorrízicos arbusculares (FMA) desempenham importante papel no ciclo de nutrientes em florestas tropicais e estão intimamente envolvidos com o estado vegetativo das plantas com as quais estão associados. Com a crescente degradação de áreas naturais nas últimas décadas, principalmente florestas, cientistas e ambientalistas têm estado cada vez mais empenhados em restabelecer a composição da flora original, como forma de recuperar o potencial genético dessas áreas (Barbosa et al. 1989; Kageyama et al. 1989).

Informações sobre a contribuição de FMA em ecossistemas desequilibrados são ainda escassas (Brundrett et al. 1996; Johnson \& Wedin 1997) apesar da necessidade urgente de se conhecer a função desempenhada por estes na dinâmica dos ecossistemas. Também, pouco é conhecido sobre a relação existente entre FMA e sucessão de espécies de plantas nativas em ecossistemas naturais (Janos 1980; Carrenho et al. 1997; Zangaro Filho 1997; Siqueira et al. 1998), sendo os dados geralmente conflitantes.

A perda de Matas ciliares tem sido motivo de preocupação nas últimas décadas, pois estas garantem a estabilidade das áreas que margeiam os rios, evitando o assoreamento de reservatórios, a erosão e o empobrecimento do solo, que, por sua vez, ocasionam redução da biodiversidade local.

Programas de recuperação de matas ciliares têm sido realizados a partir do plantio de espécies nativas, de diferentes estádios serais, cultivadas em viveiros e transplantadas para campo. Para Saggin-Jr (1997), o sucesso de reflorestamentos com espécies nativas depende da capacidade de estabelecer espécies vegetais sob os estresses impostos pelo ambiente, de modo que a mata formada seja capaz de aumentar a matéria orgânica e a atividade biológica do solo, promover a ciclagem de nutrientes e iniciar o processo de sucessão. Além da dispersão natural, a diversidade em matas regeneradas dependerá diretamente da capacidade de as espécies arbóreas suportarem os estresses ambientais, nutrirem-se satisfatoriamente e adaptarem-se ao ecossistema regenerado, vivendo em competição ou sinergismo com outras espécies. Assim, o conhecimento da capacidade das espécies nativas em formar micorrizas e de se beneficiar das mesmas, torna-se essencial quando se pretende ter sucesso na regeneração de matas.

O objetivo deste estudo foi determinar a ocorrência de FMA nas rizosferas de três espécies de plantas hospedeiras (Croton urucurana Baill., Inga striata Willd. e Genipa americana L.), de diferentes estádios serais, cultivadas em área de mata ciliar revegetada.

\section{Material e métodos}

Este estudo foi desenvolvido em outubro de 1996, nas margens do rio Moji-Guaçu, município do mesmo nome, SP $\left(22^{\circ} 18^{\prime} \mathrm{S}\right.$ e $\left.47^{\circ} 11^{\prime} \mathrm{W}\right)$, com altitude ao redor dos $680 \mathrm{~m}$, onde estavam sendo desenvolvidos projetos de implementação e análise de modelos para manutenção e recuperação de áreas degradadas ao longo de rios. $\mathrm{O}$ clima na região é do tipo Cwa (classificação de Köppen), com temperaturas médias variando de $18^{\circ} \mathrm{C}$, no mês mais frio (junho), a $22^{\circ} \mathrm{C}$, no mês mais quente (fevereiro). O solo local é do tipo latossolo vermelho-amarelo, de textura arenosa e com as seguintes características químicas e físico-químicas: $\mathrm{pH}\left(\mathrm{CaCl}_{2}\right)=4,3, \mathrm{MO}=24 \mathrm{~g} \cdot \mathrm{Kg}^{-1}$, $\mathrm{P}($ resina $)=3 \mathrm{mg} \cdot \mathrm{dm}^{-3}$, e em mmolc. $\mathrm{dm}^{-3}: \mathrm{K}^{+}=$ 
$0,7, \mathrm{Ca}^{+2}=6,9, \mathrm{Mg}^{+2}=2,5, \mathrm{H}^{+}+\mathrm{Al}^{+3}=58$.

$\mathrm{A}$ área experimental possui cerca de $2.5 \mathrm{ha}$ e 2.750 indivíduos de 13 espécies típicas de mata ciliar, que haviam sido transplantados anteriormente (dezembro/1987).

A recomposição desta área foi feita a partir de uma mistura de espécies pioneiras, secundárias (inicial e tardia) e clímax, entre elas Croton urucurana (pioneira), Inga striata (secundária inicial) e Genipa americana (clímax) (Kageyama et al. 1989). Estas plantas são comumente verificadas nas margens de rios do estado de São Paulo e também apresentam elevada capacidade de sobrevivência em condições de submersão ( $C$. urucurana $93,41 \%$, G. americana $92,5 \%$ e I. striata $92,28 \%$ ), como verificado por Barbosa et al. (1989). Por essas características, foram escolhidas como plantas-alvo no presente estudo. Todas as plantas estavam com 1 a 2 anos quando transplantadas para o campo; assim quando a coleta foi realizada, elas estavam com aproximadamente 12 anos.

Amostras de solo foram coletadas de rizosferas ( $15 \mathrm{~cm}$ de profundidade) de cinco espécimes de $C$. urucurana, G. americana e $I$. striata, perfazendo um total de 15 amostras (cada uma com aproximadamente $500 \mathrm{~g}$ ). A coleta foi realizada somente em espécimes que não apresentavam outras plantas crescendo nas proximidades de suas rizosferas $(50 \mathrm{~cm}$ da base do tronco). De cada amostra foram retiradas três subamostras de $100 \mathrm{~g}$, que foram processadas de acordo com Gerdemann \& Nicolson (1963) e Jenkins (1964) com o objetivo de isolar esporos de FMA.

Esporos provenientes de cada amostra foram contados e isolados sob microscópio estereoscópico, preservados em lâminas permanentes com resina PVLG (Morton et al. 1993) e identificados com auxílio de Schenck \& Pérez (1988), Trufem (1988), Wu \& Liu (1995), além de publicações específicas mais recentes.

O número total de esporos (NT) representa o número absoluto de esporos de FMA em 100g de solo seco. O número específico de esporos
(NE) refere-se ao número de esporos de FMA produzidos por cada espécie.

A frequiência relativa de ocorrência de cada espécie foi determinada pela expressão $\mathrm{FR}=$ $\left(\mathrm{NA}^{\mathrm{T}} \mathrm{T}^{-1}\right) .100$, onde NA representa o número de amostras em que cada espécie de FMA ocorreu e TA, o número total de amostras analisadas.

A riqueza de espécies foi avaliada pelo número de espécies presentes em $100 \mathrm{~g}$ de solo seco. Dominância e diversidade foram estimadas a partir dos índices de Simpson (Simpson 1949), e equabilidade foi avaliada pelo índice J' (Pielou 1975), expressos a seguir:

Dominância: $\mathrm{C}=\Sigma\left(\mathrm{x}_{\mathrm{i}} / \mathrm{x}_{0}\right)^{2}$

onde $\mathrm{x}_{\mathrm{i}}$ é o número de esporos de uma determinada espécie $\mathrm{e} \mathrm{x}_{0}$ é $\mathrm{o}$ número total de esporos.

Equabilidade: J' = H'/H'max

onde H' é o índice de Shannon, H' max corresponde a $\log \mathrm{S}$ e $\mathrm{S}$ é o número total de espécies numa comunidade amostrada.

Diversidade: $\mathrm{H}^{\prime}=\sum_{i=1}^{\mathrm{s}} \rho i \log _{\mathrm{n}} \rho$,

onde $\rho i$ corresponde ao número de esporos de determinada espécie e $\rho$ é o número total de esporos.

\section{Resultados e discussão}

Número de esporos - Os números totais de esporos de FMA foram 85, 212 e 511 em 100g de solo das rizosferas de C. urucurana, I. striata e $G$. americana, respectivamente (Tab. 1).

Nas fases iniciais da implantação do projeto de recuperação da área por transplantio de mudas (1991), o número de esporos era menor que o verificado neste levantamento, variando de 6 a 29/100g solo (Carrenho et al. 1997). É possível que o maior número de esporos obtido neste segundo levantamento esteja relacionado com o aumento da biomassa radical, que forneceu maiores possibilidades de infecção, colonização radical e expansão micelial no solo 
Tabela 1. Número específico de esporos (NE) e freqüência relativa de ocorrência (FR) de fungos micorrízicos arbusculares em solo rizosférico (100g) de três espécies arbóreas estabelecidas em área de mata ciliar recuperada (Moji-Guaçu, SP).

\begin{tabular}{|c|c|c|c|c|c|c|}
\hline \multirow[t]{2}{*}{ Espécies de FMA } & \multicolumn{2}{|c|}{ Croton } & \multicolumn{2}{|c|}{ Inga } & \multicolumn{2}{|c|}{ Genipa } \\
\hline & $\mathrm{NE}$ & FR & $\mathrm{NE}$ & FR & $\mathrm{NE}$ & FR \\
\hline Acaulospora gerdemanni Schenck \& Nicol. & 1 & 20 & 1 & 20 & - & - \\
\hline Acaulospora longula Spain \& Schenck & - & - & - & - & 21 & 80 \\
\hline Acaulospora mellea Spain \& Schenck & 2 & 20 & 1 & 20 & - & - \\
\hline Acaulospora scrobiculata Trappe & - & - & 4 & 40 & - & - \\
\hline Acaulospora spinosa Walker \& Trappe & 4 & 20 & 20 & 80 & - & - \\
\hline Acaulospora tuberculata Janos \& Trappe & 9 & 40 & 46 & 80 & 77 & 80 \\
\hline Entrophospora kentinensis $\mathrm{Wu} \& \mathrm{Liu}$ & - & - & 25 & 100 & 28 & 80 \\
\hline Gigaspora margarita Becker \& Hall & - & - & 2 & 20 & - & - \\
\hline Glomus claroideum Schenck \& Smith & 13 & 60 & 2 & 20 & 42 & 100 \\
\hline Glomus clarum Nicol. \& Schenck & 3 & 20 & - & - & 7 & 40 \\
\hline Glomus etunicatum Becker \& Gerd. & - & - & 27 & 100 & 21 & 100 \\
\hline Glomus globiferum Koske \& Walker & 3 & 20 & - & - & - & - \\
\hline Glomus invermaium Hall & - & - & 38 & 80 & 91 & 80 \\
\hline Glomus macrocarpum Tul. \& Tul. & 28 & 100 & 25 & 100 & 35 & 100 \\
\hline Glomus microaggregatum Koske, Gemma \& Olexia & 3 & 20 & - & - & - & - \\
\hline Glomus microcarpum Gerd. \& Trappe & 5 & 20 & 5 & 20 & - & - \\
\hline Glomus occultum Walker & 7 & 40 & 4 & 20 & 161 & 100 \\
\hline Glomus sp. & - & - & - & - & 28 & 40 \\
\hline Scutellospora calospora (Nicol. \& Gerd.) Walker \& Sanders & 1 & 20 & - & - & - & - \\
\hline Scutellospora erythropa (Koske \& Walker) Walker \& Sanders & 2 & 20 & 11 & 40 & - & - \\
\hline Scutellospora fulgida Koske \& Walker & 1 & 20 & - & - & - & - \\
\hline Scutellospora heterogama Nicol. \& Gerd. & 3 & 20 & 1 & 20 & - & - \\
\hline Número total de esporos & 85 & & 212 & & 511 & \\
\hline Riqueza & 15 & & 15 & & 10 & \\
\hline Dominância & 0,16 & & 0,14 & & 0,17 & \\
\hline Diversidade & 0,84 & & 0,85 & & 0,82 & \\
\hline Equabilidade & 0,59 & & 0,37 & & 0,37 & \\
\hline
\end{tabular}

Freqüência relativa expressa em percentagem; $(n=5)$.

rizosférico. O aumento da biomassa fúngica dentro e fora das raízes junto com alterações das atividades metabólicas das plantas, como produção aumentada de fotoassimilados e maior direcionamento destes para as raízes, podem ter sustentado a maior produção de esporos. Brundrett et al. (1996) verificaram que o número de propágulos de FMA em áreas colonizadas basicamente por vegetação herbácea esparsa era muito menor que aquele detectado em áreas ocupadas principalmente por espécies arbóreas, densamente agrupadas.

A esporulação dos FMA foi relacionada ao estádio seral de cada uma das plantas estuda- das. A rizosfera da espécie pioneira ( $C$. urucurana) apresentou o menor número de esporos de FMA, e este foi aumentando progressivamente até a planta clímax ( $G$. americana) (Tab. 1).

Trabalhos realizados por Zangaro Filho (1997) e Siqueira et al. (1998) demonstraram que dependência e responsividade à associação micorrízica em mudas de espécies arbóreas nativas da Bacia do Rio Tibagi (Paraná) e do Sudeste brasileiro, respectivamente, eram maiores naquelas de estádios iniciais de sucessão e diminuíam nas clímax, e este comportamento pareceu relacionado com o tamanho das sementes 
e a quantidade de reservas nelas contidas, à produção de raízes e pêlos radicais e à demanda por fósforo. Seguindo este raciocínio, pode-se explicar o comportamento da esporulação, no presente estudo, admitindo-se que, à medida em que o volume de raízes aumenta (de pioneira à clímax), aumentam as possibilidades de "iscagem" dos FMA, uma vez que existe quantidade maior de raízes em contato com os propágulos destes organismos. Como espécies clímax parecem necessitar menos da associação com FMA (Zangaro Filho 1997; Siqueira et al. 1998), ao menos em seus estádios iniciais de crescimento e contrariando os dados obtidos anteriormente por Janos (1980), o desenvolvimento intraradical destes fungos tende a ser limitado, sendo a percentagem de colonização geralmente baixa (Zangaro Filho 1997, Siqueira et al. 1998). Com isso, pode haver dreno mais pronunciado, para o micélio extraradical, de compostos orgânicos que podem ser utilizados na formação de esporos e/ou outras estruturas, aumentando o número de propágulos infectivos no solo (Douds \& Schenck 1990). Provavelmente, nas plantas pioneiras, a principal forma de propagação destes fungos seja através de fragmentos de raízes colonizadas e/ou da conexão do micélio entre plantas vizinhas.

Diversidade de FMA - Foram identificadas 22 espécies de FMA pertencentes a cinco gêneros: Acaulospora (6), Entrophospora (1), Gigaspora (1), Glomus(10) e Scutellospora (4).

Em levantamento anterior, realizado na mesma área (Carrenho et al. 1997), foram isoladas somente oito espécies. Percebe-se que, à medida que as plantas cresceram e a área foi sendo colonizada, as condições ambientais melhoraram e permitiram a instalação de maior número de espécies potencialmente hospedeiras que, por sua vez, permitiram o aumento da riqueza da comunidade de FMA. Lodge \& Cantrell (1995) verificaram que florestas com altura e complexidade estrutural mais elevadas criam mais microhabitats e microclimas, favo- recendo a diversificação dos microrganismos do solo. Em Moji-Guaçu, além das 13 espécies arbóreas transplantadas na área objeto deste estudo, pode-se encontrar muitas outras, de diferentes hábitos (trepadeiras, rasteiras, herbáceas, arbustivas) que se instalaram depois, via dispersão de sementes e/ou diasporos produzidos nas áreas vizinhas. Esse aumento de diversidade vegetal pode ter colaborado para a riqueza de espécies de FMA. Por outro lado, alguns estudos recentes vêm demonstrando que quando a riqueza de espécies de FMA é aumentada, também são aumentados a diversidade de plantas, a captura de nutrientes minerais e a produtividade dos ecossistemas (van der Heidjen et al., 1998).

Habitats que sofrem modificações (estresses) periódicas do ambiente, como por exemplo áreas que são alagadas nas beiras dos rios, parecem receber "invasores" muito facilmente (Hanski et al. 1993). Esporos e/ou outros propágulos de FMA de uma região podem ser levados pela correnteza dos rios e se instalar em outras. Outros agentes dispersores (vento, aves, roedores etc.) podem também ter colaborado para a disseminação destes fungos, contribuindo, assim, para o aumento da diversidade de espécies nestas áreas.

Em áreas contíguas, com vegetação de cerrado nativo e protegido (Bononi \& Trufem 1983) ou onde foram introduzidas diversas culturas (Trufem \& Bononi 1985), registrou-se a ocorrência de 24 espécies de FMA. Destas, seis foram verificadas no presente levantamento: Acaulospora scrobiculata, A. spinosa, Glomus macrocarpum, G. microcarpum, Scutellospora calospora e $S$. heterogama, fato que sugere elevada dispersão, adaptabilidade e persistência destas espécies nas condições ambientais da região.

A capacidade de esporulação dos FMA pode variar com a planta hospedeira assim como com características edáficas (Brundrett 1991; Douds 1994). Observou-se que em cada planta foi estabelecida uma comunidade diferente de 
FMA. Esta seleção aparentemente mediada pela planta hospedeira pode ser resultante do estabelecimento de associações preferenciais entre os participantes micorrízicos. Isto pode depender de características morfológicas e fisiológicas da planta, assim como da compatibilidade genética entre planta e fungo (Smith 1995) que junto com os fatores ambientais, definem a especificidade entre os simbiontes (Mc Gonigle \& Fitter 1990).

Em rizosferas de $C$. urucurana registrou-se a ocorrência de 15 espécies de FMA (Tab. 1), sendo Glomus macrocarpum a mais abundante (32,9\% dos esporos) e freqüente $(100 \%$ das amostras), seguida por Glomus claroideum (15,3\% de abundância e $60 \%$ de frequiência). Das 15 espécies isoladas, 11 foram consideradas raras por ocorrerem em até $20 \%$ das amostras de solo e duas (A. tuberculata e $G$. occultum) apresentaram baixa frequiência de ocorrência (40\%). Essa baixa representatividade da maioria das espécies pode indicar que somente algumas espécies estavam colonizando as raízes de Croton na época da coleta. É possível que as muitas substâncias com propriedades antibióticas produzidas por plantas deste gênero, inclusive $C$. urucurana (Ramesh et al. 1995; Peres et al. 1997), tenham inibido a germinação de esporos e a infecção radical de muitas espécies de FMA, sendo G. macrocarpum e G. claroideum menos afetados. Assim, estas espécies podem ter se estabelecido dentro das raízes e em momento oportuno, esporulado. No entanto, não podemos afirmar que este tenha sido um fator determinante na composição de espécies de FMA, uma vez que não avaliamos a presença e a influência destes compostos sobre o estabelecimento da associação micorrízica, nem determinamos quais espécies estavam efetivamente associadas às raízes de $C$. urucurana.

Espécies raras ou de baixa freqüência podem estar presentes no ambiente sob outras formas (células auxiliares, hifas, raízes colonizadas) ou simplesmente aparecerem como resquício de uma comunidade pré-estabelecida em planta de ciclo-de-vida curto, ou ainda terem sido produzidas nas proximidades e dispersadas sem obterem êxito na ocupação do novo ambiente. Os outros propágulos fúngicos são menos tolerantes aos estresses ambientais que os esporos e podem desaparecer mais rapidamente (de Souza \& Silva 1996). Assim, algumas espécies podem ter sido excluídas no processo de competição. Porém, a ausência de esporos não indica, necessariamente, ausência de colonização radical e, consequentemente, não participação na associação simbiótica.

Diferenças na ontogenia e morfologia (cor, tamanho, estrutura e espessura das paredes) podem ter contribuído para o sucesso de espécies como G. macrocarpume G. claroideum. Esporos pequenos tendem a ser carregados com a água da chuva, para as porções mais profundas do solo, sendo assim protegidos do ataque de parasitas e predadores que povoam as regiões mais superficiais do solo, onde a deposição de matéria orgânica é maior. Esporos formados apicalmente em uma hifa esporígena (Glomus, Gigaspora e Scutellospora) apresentam, desde o início de sua formação, parede mais espessada e resistente que aqueles formados a partir de vesículas (Acaulospora e Entrophospora). Além disso, velocidade de germinação, formação de hifas e infecção são características importantes no processo de competição inter-específica. Assim, G. macrocarpum pode apresentar maior resistência ao ataque de microrganismos e predadores, e também aos estresses edáficos, e persistir por mais tempo no solo. Com estas características, pode efetuar constante re-infecção radical e com o passar de tempo, se outras espécies não se estabelecerem, G. macrocarpum pode tornar-se dominante.

Espécies de Glomineae (7 de Glomus e 4 de Acaulospora) predominaram e somente 4 espécies de Gigasporineae (todas Scutellospora) foram isoladas. Observou-se ainda elevada equabilidade $(0,59)$ na comunidade de FMA formada nas rizosferas de Croton. Scutellospora calospora, S. fulgida, Glomus globiferum e $G$. 
microaggregatum foram encontrados exclusivamente em rizosferas desta planta pioneira. Porém, não se pode afirmar que este seja um caso de associação preferencial, pois os números de esporos eram muito baixos $(1,1,3$ e 3 , respectivamente). Estas espécies podiam estar presentes na comunidade acidentalmente e não como espécies estabelecidas de fato. No entanto, não se pode descartar a possibilidade de estarem presentes na raiz, sem a produção de esporos.

Em rizosferas de 1 . striata também foram isoladas 15 espécies de FMA, sendo $A$. tuberculata a mais abundante $(21,7 \%$ dos esporos) seguida por Glomus invermaium (17,9\%) (Tab. 1). A. tuberculata também havia sido dominante, em termos de número de esporos, em levantamento anterior (Carrenho et al. 1997). Parece haver, neste caso, preferência desta espécie pela planta hospedeira, assim como esta ser boa competidora, pois manteve a dominância dentro da comunidade de FMA, mesmo em diferentes fases de crescimento de Inga.

Espécies com elevado número de esporos, e estes sendo encontrados em diferentes etapas de desenvolvimento, confirmam a condição micotrófica e podem estar participando mais efetivamente da colonização radical. Segundo dados obtidos por Zangaro Filho (1997), Siqueira et al. (1998) e Carrenho et al. (1997) a percentagem de colonização em plantas secundárias (inclusive I. striata) é mais baixa que aquela obtida em plantas pioneiras ( $C$. urucurana, por exemplo). Com baixa colonização intraradical, as raízes passam a ter menor importância como fonte de inóculo, o que aumenta a importância dos esporos, principalmente daqueles mais resistentes e, consequentemente, persistentes. Kabir et al. (1996) observaram que a percentagem de CR esteve positivamente relacionada com a abundância de hifas no solo, reforçando a idéia de que hifas extraradicais podem ser a principal forma de propagação dos FMA estabelecidos em plantas com elevadas percentagens de CR e dependência micorrízica.
Verificou-se na comunidade rizosférica de Inga, riqueza similar à de Croton; porém, a comunidade de FMA nesta planta foi mais diversa (índice de Simpson $=0,85)$ principalmente em termos taxonômicos (espécies de 5 gêneros versus 3 gêneros em Croton). É provável que I. striata seja pouco seletiva podendo, portanto, ser colonizada por vários gêneros de Glomales. Além disso, o maior volume de raízes desta planta (comparado à anterior) pode ter criado mais oportunidades para o estabelecimento intraradical de diversas espécies de FMA, possibilitando assim, esporulação de maior número destas. Observou-se, também, menor número de espécies raras (sete espécies) e com baixa freqüência (duas espécies) e maior número de espécies freqüentemente presentes (seis espécies) que na comunidade de FMA estabelecida em $C$. urucurana. Assim, pôde-se constatar que a maioria das espécies estava bem representada nas amostras (em termos de abundância), porém a distribuição não foi uniforme, o que ocasionou redução de dominância $(0,14)$ e de equabilidade $(0,37)$. Duas espécies ( $A$. scrobiculata e $G$. margarita) ocorreram exclusivamente em I. striata, embora como possíveis acidentais.

Em rizosferas de $G$. americana foram isoladas dez espécies de FMA, sendo Glomus occultum a espécie mais abundante $(31,5 \%$ dos esporos) seguida por $G$. invermaium e $A$. tuberculata $(17,8 \%$ e $15 \%$, respectivamente). Em relação à frequiência, oito espécies ocorreram na maioria das amostras (80-100\%); porém, não se verificou elevada equabilidade nestas comunidades (0,37). Como $G$. occultum, $G$. invermaiume $A$. tuberculata ocorreram em maior número que as outras sete espécies, verificou-se elevado índice de dominância $(0,17)$ na comunidade de FMA desta planta. Adicionalmente, observou-se que duas espécies (Glomus sp. e $A$. longula) só ocorreram em $G$. americana.

Embora o número de amostras tenha sido pequeno e não se tenha avaliado a influência de exsudados radicais sobre os FMA, metabólitos 
secretados pelas raízes de $C$. urucurana e $I$. striata podem ter inibido a germinação dos esporos ou o crescimento das outras estruturas fúngicas com potencial infectivo, impedindo o estabelecimento destas espécies (Glomus sp. e A. longula) em suas rizosferas.

Em $G$. americana, a comunidade foi formada principalmente por espécies que apresentaram elevada distribuição (80-100\%), e somente duas espécies apresentaram baixa freqüência de ocorrência (G. clarum e Glomus sp.). A primeira provavelmente é uma espécie acidental, pelo baixo número de esporos verificado. A limitada ocorrência de Glomus sp. entre as amostras, pode ser devida a fatores como baixa capacidade competitiva e infectividade, reduzida formação de micélio extraradical, os quais podem ter ocasionado o estabelecimento pontual da espécie na rizosfera. É provável que, em condições de campo, espécies com melhores estratégias de sobrevivência tenham sido mais rápidas no processo de ocupação radical, diminuindo as chances do estabelecimento de Glomus sp. Assim, nas regiões rizosféricas onde essa espécie conseguiu se estabelecer, pôde-se verificar expressivo número de esporos.

Sabe-se que no processo de esporulação o fungo requer elevada quantidade de compostos orgânicos, principalmente lipídios, o que ocasiona acentuado dreno destes para o fungo (Peng et al. 1993). Como plantas clímax usualmente apresentam dimensões mais avantajadas, a produção de fotoassimilados tende a ser maior para sustentar o crescimento destas plantas. Com sistema radical mais desenvolvido, velocidade de crescimento mais lenta e menor dependência à micorrização (Zangaro Filho 1997; Siqueira et al. 1998), provavelmente estas plantas suportam a colonização de maior número de espécies de FMA. Assim, a comunidade de FMA em $G$. americana, embora menos rica (quando comparada àquelas verificadas em rizosferas de $C$. urucurana e I. striata), possivelmente foi formada por maior número de espécies efetivamente associadas com a raiz. Isto somado à maior disponibilidade de nutrientes orgânicos para as raízes, deve explicar o elevado número de esporos em G. americana. Também é importante ressaltar que, embora as raízes tenham suportado esporulação abundante, os gastos que esta planta teve com a associação (formação de hifas intra e extraradicais, absorção de minerais e troca de nutrientes) não devem ter sido muito grandes, uma vez que as percentagens de colonização em plantas clímax geralmente são baixas (Carrenho et al. 1997; Zangaro Filho 1997).

Nossos dados sugerem que: a) diferentes espécies de plantas hospedeiras criam um habitat próprio aos redor de suas raízes, levando ao estabelecimento de espécies distintas de FMA; b) o ritmo do crescimento das plantas, a biomassa radical e a competição por fotoassimilados parecem ser fatores limitantes da esporulação de FMA; c) a ocorrência de espécies de FMA com baixo número de esporos é mais comum nas plantas pioneira e secundária; d) o número de esporos é maior nas rizosferas de plantas dos estádios finais da sucessão; e) espécies de Glomineae esporulam mais e são mais comuns que as de Gigasporineae.

\section{Referências bibliográficas}

Barbosa, L. M.; Barbosa, J. M.; Batista, E. A.; Mantovani, W.; Veronese, S. A. \& Andreani Jr, R. 1989. Ensaios para estabelecimento de modelos para recuperação de áreas degradadas de mata ciliares, Moji Guaçu (SP) - nota prévia. Pp. 268-283. In: Anais do Simpósio sobre Mata Ciliar. Fundação Cargill, Campinas.

Bononi, V. L. R. \& Trufem, S. F. B. 1983. Endomicorrizas vesículo-arbusculares do cerrado da Reserva Biológica de Moji-Guaçu, SP, Brasil. Rickia 10: 55-84.

Brundrett, M. C. 1991. Mycorrhizas in natural ecosystems. Advances in Ecological Research 21: 131-171.

Brundrett, M. C.; Ashwath, N. \& Jasper, D. A. 1996. Mycorrhizas in the Kakadu region of tropical Australia: I. Propagules of mycorrhizal fungi and soil properties in natural habitats. Plant and Soil 184(1): 159-171. 
Carrenho, R.; Bononi, V. L. R. \& Barbosa, L. M. 1997. Glomales em áreas de recomposição de mata ciliar de Moji-Guaçu, SP, Brasil. Hoehnea 24(1): 107-113.

de Souza, F. A. \& Silva, E. M. R. 1996. Micorrizas arbusculares na revegetação de áreas degradadas. Pp. 255-290. In: J. O. Siqueira (Ed.). Avanços em fundamentos e aplicação de micorrizas. Universidade Federal de Lavras, Lavras.

Douds, D. D. 1994. Relationships between hyphal and arbuscular colonization and sporulation in mycorrhiza of Paspalum notatum. New Phytologist 126: 233-237.

Douds, D. D. \& Schenck, N. C. 1990. Relationship of colonization and sporulation by VA mycorrhizal fungi to plant nutrient and carbohydrate contents. New Phytologist 116: 621-627.

Gerdemann, J. W. \& Nicolson, T. H. 1963. Spores of mycorrhizal Endogone species, extracted from soil by wet-sieving and decanting. Transactions of the British Mycological Society 46: 235-244.

Hanski, I.; Kouki, J. \& Halkka, A. 1993. Three explanations of the positive relationship between distribution and abundance of species. Pp.108-116. In: R.E. Ricklefs \& D. Schluter (Eds.). Species diversity in ecological communities - historical and geographical perspectives. University of Chicago Press, Chicago.

Janos, D. P. 1980. Mycorrhizae influence tropical succession. Biotropica 12: 56-64.

Jenkins, W. R. 1964. A rapid centrifugal-flotation technique for separating nematodes from soil. Plant Disease Report 48: 692.

Johnson, N. C. \& Wedin, D. A. 1997. Soil carbon, nutrients, and mycorrhizae during conversion of dry tropical forest to grassland. Ecological Applications 7(1): 171-182.

Kabir, Z.; O'Halloran, I. P. \& Hamel, C. 1996. The proliferation of fungal hyphae in soils supporting mycorrhizal and non-mycorrhizal plants. Mycorrhiza 6(6): 477-480.

Kageyama, P. Y.; Castro, C. F. A \& Carpanezzi, A. A. 1989. Implantação de matas ciliares: estratégias para auxiliar a sucessão secundária. Pp. 130-143. In: Anais do Simpósio sobre Mata Ciliar. Fundação Cargill, Campinas.

Lodge, D. J. \& Cantrell, S. 1995. Fungal communities in wet tropical forests: variation in time and space. Canadian Journal of Botany (suppl.) 73:13911398.

McGonigle, T. P. \& Fitter, A. H. 1990. Ecological specificity of vesicular-arbuscular mycorrhizal associations. Mycological Research 94: 120-122.
Morton, J. B.; Bentivenga, S. P. \& Wheeler, W. W. 1993. Germ plasm in the international collection of arbuscular and vesicular-arbuscular mycorrhizal fungi (INVAM) and procedures for culture development, documentation and storage. Plant and Soil 159: 47-59.

Peng, S.; Eissenstat, D. M.; Graham, J. H. \& Williams, K. 1993. Growth depression in mycorrhizal citrus at high phosphorus supply: analysis of carbon costs. Plant Physiology 101: 1063-1071.

Peres, M. T. L. P.; Delle-Monache, F.; Cruz, A. B.; Pizzalatti, M. G. \& Yunes, R. A. 1997. Chemical composition and antimicrobial activity of Croton urucurana Baillon (Euphorbiaceae). Journal of Ethnopharmacology 56(3): 223-226.

Pielou, E. C. 1975. Ecological diversity. John Willey \& Sons, New York. 165p.

Ramesh, V. M.; Hilda, A. \& Manjula, V. K. 1995. Fungitoxic effect of leaf extract of Croton sparsiflorus Morong on phytopathogenic fungi. Acta Botanica Indica 23(1): 63-66.

Saggin-Jr, O. J. 1997. Micorrizas arbusculares em mudas de espécies arbóreas nativas do sudeste brasileiro. Tese de Doutorado. Universidade Federal de Lavras, Lavras. 120p.

Schenck, N. C. \& Pérez, Y. 1988. Manual for the identification of VA mycorrhizal fungi. IFAS, University of Florida, Gainesville. 241p.

Simpson, E. H. 1949. Measurement of diversity. Nature 163: 188.

Siqueira, J. O.; Carneiro, M. A. C.; Curi, N.; Rosado, S. C. S. \& Davide, A. C. 1998. Mycorrhizal colonization and mycotrophic growth of native wood species as related to sucessional groups in southeastern Brazil. Forest Ecology and Management 107: 241-252.

Smith, S. E. 1995. Discoveries, discussions and directions in mycorrhizal research. Pp.3-24. In: A. Varma \& B. Hock (Eds.). Mycorrhiza: structure, function, molecular biology and biotechnology. Springer Verlag, Berlin.

Trufem, S. F. B. 1988. Fungos micorrízicos vesículoarbusculares da Ilha do Cardoso, SP, Brasil. Tese de Doutorado. Instituto de Biociências, Universidade de São Paulo, São Paulo. 358p.

Trufem, S. F. B. \& Bononi, V. L. R. 1985. Micorrizas vesículo-arbusculares de culturas introduzidas em áreas de cerrado. Rickia 12: 165-187.

van der Heidjen, M. G. A.; Klironomos, J. N.; Ursic, M.; Moutoglis, P.; Streitwolf, E. R.; Boller, T.; Wiemken, A. \& Sanders I. R. 1998. Mycorrhizal fungal diversity determines plant biodiversity, ecosystem variability and productivity. Nature 396 (6706): 69-72. 
Wu, C. G. \& Liu, Y. S. 1995. Glomales of Taiwan: Glomus chimonobambusae and Entrophospora kentinensis, spp. nov. Mycotaxon 53: 283-294.
Zangaro Filho, W. 1997. Micorrizas arbusculares em espécies arbóreas nativas da Bacia do Rio Tibagi (PR) e suas relações com os grupos sucessionais. Tese de Doutorado. Universidade de São Paulo, São Paulo. 155p. 